

Jourmal of

Nmitrition and Food Security

Shahid Sadoughi University of Medical Sciences

School of Public Health

Deppartment of Nutution

Naturition \& Food Security Research Center

pISSN: 2476-7417

JNFS 2019; 4(1): 58-65

Website: jnfs.ssu.ac.ir

\title{
Polycystic Ovary Syndrome and Dietary Patterns in Iran: A Review Study
}

Asieh Panjeshahin; BSc ${ }^{1,2}$, Maryam Khosravi; $\mathrm{PhD}^{3,4}$ \&Mahdieh Hosseinzadeh: PhD ${ }^{* 1,2}$

${ }^{1}$ Nutrition and Food Security Research Center, Shahid Sadoughi University of Medical Sciences, Yazd.

${ }^{2}$ Department of Nutrition, School of Public Health, Shahid Sadoughi University of Medical Sciences, Yazd, Iran.

${ }^{3}$ Department of Nutrition, School of Medicine, Mashhad University of Medical Sciences, Mashhad, Iran.

${ }^{4}$ Department of Public Health, North Khorasan University of Medical Sciences, Bojnurd, Iran.

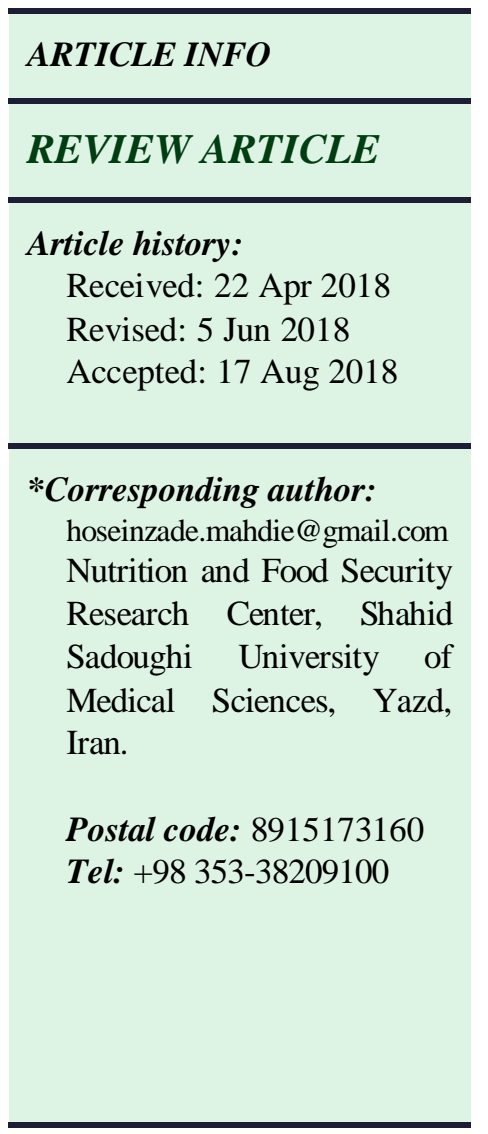

\section{ABSTRACT}

Background: Polycystic Ovary Syndrome (PCOs) is one of the most common metabolic and endocrine abnormalities among women in reproductive age. In the case of not comprehensive treatment, PCOs can lead to hormonal, metabolic, and fertility disorders. The exact cause of PCOs is still unclear. This disease seems to have a genetic background caused by the interference of several key genes with the environmental factors such as dietary habit and food intake, which play an important role in prevention and treatment of this syndrome. Methods: We searched Scopus, PubMed, SID, and Magiran data bases to find the studies conducted in Iran on dietary patterns, dietary intake, food intake, and PCOs published in English. Results: The findings showed that decrease of weight and fat intake from total calorie (especially saturated fat and cholesterol), increase of physical activity and intake of dietary fibers can improve this syndrome. Furthermore, DASH diet, increase of the protein/carbohydrate ratio in the diet, the low-calorie diets, or iso-caloric diets with a low glycemic index can also be useful in this regard. Discussion: In recent studies, the effects of some healthy diets were studied on PCOs women. A few of these studies were about finding appropriate dietary patterns for PCOs patients, but their number was limited. So, further studies are needed in this regard.

Keywords: PCOs; Dietary pattern; DASH diet; Oxidative stress; Visceral adiposity.

\section{Introduction}

Dolycystic Ovary Syndrome (PCOs) is a multifactorial and multigenic disorder. It is also one of the most common metabolic and endocrine abnormalities among women in reproductive age, with a prevalence of 7-15 percent in Iran (Azadi-Yazdi et al., 2017). The
National Institute of Child Health and Human Disease (NIH)) as well as Rotterdam and Androgen Excess Society (AES) are commonly used to evaluate the prevalence of PCOs. However, Rotterdam is more common today (Jalilian et al., 2015). Diagnostic criteria suggested by Rotterdam 
include the presence of two of the three diagnostic criteria:

- Oligomenorrhea or amenorrhea associated with decreased ovulation. The most common cause of anovulatory infertility is PCOs.

- Hyperandrogenaemia or clinical features of androgen excess, in the absence of other underlying disease states.

- Abnormal ovarian ultrasound with $\geq 12$ follicles in each ovary having a diameter of $2-9$ $\mathrm{mm}$, or increased ovarian volume (Farshchi et al., 2007).

In the absence of treatment, PCOs can cause fertility problems such as infertility, impaired hemorrhage, endometrial carcinoma, depression, low self-confidence, anxiety and sleep apnea, sexual function abnormalities, metabolic disorders such as obesity, Impaired glucose tolerance, type 2 diabetes, hypertension and, and hypertension (Goodarzi et al., 2011, Palomba et al., 2015, Pastore et al., 2011, Wang et al., 2011). It also can lead to abortion by increasing levels of LH, insulin, obesity, as well as increased risk of gestational diabetes mellitus (GDM), lipid profiles, and biomarkers of oxidative stress. One of the common characteristics of PCOs is obesity. Many studies reported that 5-10 percent weight loss can reduce the risk of cardiovascular diseases, type 2 diabetes, endocrine, and reproductive parameters in PCOs women (Faghfoori et al., 2017).

Commonly used treatments include consumption of clomiphene, oral contraceptives, gonadotropins, GnRH agonists, co-utilization of vitamin D and calcium, as well as use of weight loss diets. In resistant cases, laparoscopy is required (Carmina et al., 2016, Cree-Green, 2017, Kalem et al., 2017). Insulin resistance is a major factor in pathogenesis of PCOs (Palomba et al., 2015). Therefore, extensive use of insulin lowering drugs such as metformin is recommended to treat this syndrome. Furthermore, recent studies indicated that some minerals can have positive effects on PCOs or complications of this syndrome: positive effects of chromium on insulin sensitivity (Amr and Abdel-Rahim, 2015), magnesium and selenium on serum testosterone level, as well as double bond unsaturated fatty acids such as omega- 3 on risk factors of metabolic syndrome (Faghfoori et al., 2017). Many studies recommended decrease of weight, intake of fat from total calories (especially saturated fat and cholesterol), increase of physical activity, intake of dietary fibers, and change of diets. The investigated diets to improve this syndrome included increase of the protein/carbohydrate ratio (Sorensen et al., 2012), intake of high protein and low glycemic load (LGL) foods, low glycemic index (LGI) diets, low calorie diets (Love et al., 2016), and an iso-caloric diet with a LGI. In recent years, some researches were conducted over the healthy dietary patterns in women with PCOs. A few of these studies tried to find appropriate dietary patterns for this syndrome. In the present research, we only included the studies appropriate on dietary patterns for women with PCOs conducted over recent years in Iran.

\section{Materials and Methods}

We searched the databases of PubMed, Scopus, ISI Web of Science, SID, Magiran, and Google scholar to find the studies written in English conducted on dietary patterns, dietary intake, food intake, and PCOs in Iran. We searched the studies published up to June 2017.

In order to conduct the search, we used the title/abstract, $\mathrm{MeSH}$ terms, and other related key words including (dietary pattern OR dietary intake) AND (polycystic ovary syndrome OR PCOs) as well as other related words. In addition to the mentioned databases, we conducted manual search over all eligible original articles, reviews, and other relevant articles.

\section{Results}

Table 1 shows the descriptive data of all seven studies. In the following sections, we represent the studies conducted over each category of subjects. Dietary approach for stopped hypertension (DASH) diet and weight loss, anti-mullerian hormone (AMH), metabolic profiles, androgens, body composition, and antioxidant effect in women with PCOs.

A randomized clinical trial study was designed 
to evaluate the effects of the DASH diet on weight loss, AMH, and metabolic profiles in women with PCOs (Foroozanfard et al., 2017). To this point, 60 overweight or obese women with PCOs were studies; 30 participants were in DASH diet group and 30 in control group. The DASH and control diets consisted of 52-55 percent carbohydrates, 1618 percent proteins, and 30 percent total fats. The DASH diet was rich in fruits, vegetables, whole grains, low-fat, low cholesterol dairy products, refined grains, and sodium levels less than 2400 mg per day. The amounts of calories' intake were equal in both groups. These results were obtained after 12 weeks: participants had significant reduction in body mass index (BMI) $(1.6 \pm 0.5 \mathrm{vs}$. $\left.-1.2 \pm 0.7 \mathrm{~kg} / \mathrm{m}^{2}, P=0.02\right)$, AMH $(-1.1 \pm 3.1 \mathrm{vs}$. $0.3 \pm 0.7 \mathrm{ng} / \mathrm{mL}, P=0.01)$, malondialdehyde (MDA) $(-0.5 \pm 0.4$ vs. $0.2 \pm 0.3 \mu \mathrm{mol} / \mathrm{L}, P<$ 0.001), homeostasis model of assessmentestimated insulin resistance HOMA-IR, HOMA-B $(-0.9 \pm 2.0$ vs. $-0.1 \pm 1.0, P=0.02)$, and free androgen index (FAI) $(-0.03 \pm 0.09$ vs. $0.06 \pm$ $0.21, P=0.02)$. However, the DASH group had significant increase in quantitative insulin sensitivity check index $(0.01 \pm 0.03$ vs. $0.004 \pm$ $0.01, P=0.02$ ), sex hormone-binding globulin (SHBG) $(3.7 \pm 8.5$ vs. $-1.5 \pm 7.2 \mathrm{nmol} / \mathrm{L}, P=$ $0.01)$, and nitric oxide (NO) $(9.0 \pm 4.9$ vs. $0.6 \pm 2.3$ $\mu \mathrm{mol} / \mathrm{L}, P<0.001)$ compared with the control group. After adjustment for the baseline values of biochemical variable, BMI, and age, the findings were not influenced.

The other study was a randomized controlled trial over DASH diet, androgens, antioxidant status, and body composition in overweight and obese women with PCOs (Azadi-Yazdi et al., 2017). In a period of 3 months, PCOs was diagnosed in 60 women with overweight or obesity aged 20-40 years (BMI: 25-40) according to the Rotterdam criteria. Anthropometric characteristics, dietary intake, and serum androgens were measured at the beginning and at the end of the study. Initial results showed differences in testosterone and the secondary outcomes included changes in androstenedione, (SHBG: Sex hormone-binding globulin), total antioxidant capacity, and body composition. The combination of macronutrients was similar in interventional and control groups, containing 50-55 percent carbohydrates, $15-20$ percent protein, and 25-30 percent total fat. People were asked to write their 3-day food recall (two days of week days and one day in weekend) per month. The comparison between the two groups indicated a significant decrease in BMI, body mass, and serum androstenedione. Furthermore, concentrations of SHBG and free androgen index and 2,2'-diphenyl1-picryylhydrazyl (DPPH) scavenging activity increased in the DASH group compared to the control group. After adjusting for the potential confounding variables, the researcher reported that these differences were not significant.

Therefore, we can conclude that: DASH diet have high amount of antioxidant content and this dietary pattern may have beneficial effects on reduction of BMI, MDA, insulin resistance, free androgen index (FAI), and serum androstenedione. It also can have incremental effects on insulin sensitivity, SHBG, and NO in women with PCOs.

As mentioned before, DASH diet contains high amounts of total dietary fiber, phytoestrogens, antioxidants, isoflavones, magnesium, and calcium that have good effect on oxidative stress and serum hs-CRP. Moreover, intake of both antioxidants and magnesium was inversely associated with insulin resistance. Two studies were conducted in this regard:

Overweight or obese women with $\mathrm{BMI} \geq 25$ $\mathrm{kg} / \mathrm{m}^{2}$ in the age range of $18-40$ years were diagnosed with PCOs according to the Rotterdam criteria.

The selected participants were categorized into two groups: 24 in the DASH diet and 24 in the control group. The DASH and control diets consisted of 52 percent carbohydrates, and 18 percent proteins, 30 percent total fats. . As a result, the researchers found a significant decrease in participants' weight $(4.4$ vs. $-1.5 \mathrm{~kg} ; P<0.001)$, BMI $\left(-1.7\right.$ vs. $\left.-0.6 \mathrm{~kg} / \mathrm{m}^{2} ; P<0.001\right)$, serum triglyceride (TG) $(-10.0$ vs. $+19.2 \mathrm{mg} / \mathrm{dL}$; $P=0.005)$, and VLDL-c levels $(-2.0$ vs. 3.9 $\mathrm{mg} / \mathrm{dL} ; P=0.005)$. However, the concentrations of 
total antioxidant capacity (TAC) (+98.6 vs. 174.8 $\mathrm{mmol} / \mathrm{L} ; P<0.001)$ and total glutathione $(\mathrm{GSH})$ (+66.4 vs. $-155.6 \mu \mathrm{mol} / \mathrm{L} ; P=0.005)$ increased in the DASH group compared with the control group. After adjustment for weight, TG $(-5.9 \pm 7.5$ vs. $15.2 \pm 7.5 \mathrm{mg} / \mathrm{dL}, P=0.07)$ and VLDL-c levels ($1.2 \pm 1.5$ vs. $3.0 \pm 1.5 \mathrm{mg} / \mathrm{dL}, P=0.07)$ reduced in the DASH group (Asemi et al., 2014).

Regarding the insulin resistance and serum hsCRP, the study showed a significant reduction in serum insulin levels $(-1.88$ vs $.2 .89 \mu \mathrm{IU} / \mathrm{mL}, P=$ $0.03)$, HOMA-IR score ( -0.45 vs. $0.80 ; P=0.01)$, and serum hs-CRP levels ( -763.29 vs. 665.95 $\mathrm{ng} / \mathrm{mL}, P=0.009)$ in DASH group. Furthermore, the waist size $(-5.2$ vs. $-2.1 \mathrm{~cm} ; P=0.003)$ and hip circumference (-5.9 vs. $-1 \mathrm{~cm} ; P<0.0001)$ reduced in DASH group compared with the control group. No significant differences were observed in the mean changes of fasting plasma glucose and HOMA-B between the two groups. After adjusting for age and the baseline weight measure, no significant changes were reported in the findings. After adjustment for the baseline values of biomarkers, a significant difference was seen in quantitative insulin sensitivity check index (QUICKI) between the two groups ( 0.02 vs. -0.01 , $P=0.04$ ) (Asemi and Esmaillzadeh, 2015).

So, we found that the DASH diet could be an appropriate dietary pattern for women with PCOs. In recent years, some studies were conducted to evaluate and compare the dietary intakes of women with PCOs and healthy women. These studies also determined the relationship of the dietary patterns with cardiometabolic risk factors in women with PCOs in Iran.

Dietary intake in PCOs and healthy women: To achieve the difference between the dietary intake of PCOs and healthy women, a case-control study was conducted on 142 women with PCOs and 140 eumenorrheic non hirsute women (age and BMI were matched between the two study groups). In this study, the dietary intakes of the two groups were compared using a validated Food Frequency Questionnaire (FFQ). The findings showed that energy and macronutrient intakes were almost the same between the women with PCOs and the controls. Women with PCOs had significantly higher weight measures (case: $69.37 \pm 14.97$, control: $67.45 \pm 12.92, P=0.048)$ and hip circumferences (case: $104.9 \pm 10.1$, control: 101.5 $\pm 10.73, P=0.007)$, but they had lower waist/hip ratio in comparison with the control group (case: $0.80 \pm 0.73$, control: $0.83 \pm 0.09, P=0.031)$. Consumption of high GI food items and egg in PCOs women was significantly higher than the controls, but intakes of vegetable and legume were significantly lower in PCOs women $(P<0.05)$ (Shishehgar et al., 2016).

Anthropometric characteristics and dietary patterns in women with PCOs: Recently, the researchers concluded that the dietary intake of women with PCOs should be determined. Therefore, a case-control study was conducted on 65 women with PCOs and 65 age-matched healthy women in 2013. Demographic, anthropometric, and dietary intake data were collected from each group and the results were compared. No significant difference was observed between the mean of the BMI of the two groups, but in PCOs group the mean of waist circumference was significantly higher than the control group $(P=$ 0.016). Consumption of calories and fat was significantly higher In PCOs women and the frequency of overweight patients with hirsutism was higher in PCOs group (Ahmadi et al., 2013).

This study was only about the food and dietary intakes in women with PCOs compared with the healthy women. So, it did not investigate the dietary patterns.

Dietary pattern and cardiometabolic risk factors in women with PCOs: A case- control study was conducted on 53 women with PCOs and 167 agematched healthy women in 2017. The participants were selected from the population of Tehran lipid and glucose study. Dietary intakes were collected using a semi-quantitative FFQ. The relationship between the dietary patterns and cardiometabolic risks was investigated by Pearson's correlation and linear regression. The dietary pattern was characterized by high consumption of fried 
vegetables, vegetable oils (except olive oil), salty snacks, legumes, eggs, fast foods, onion, and garlic. However, it was defined with low intakes of traditional sweets, high fat dairy, low fat dairy, cruciferous vegetables, sugars, and honey, which had positive correlation with visceral adiposity index (VAI). Consumption of this dietary pattern had a significant association with higher TG and TG/HDL-c ratio as well as lower HDL-c in both groups. A positive correlation was also found for systolic blood pressure, waist circumference, total cholesterol (TC), and LDL-c in the control group. After adjusting for BMI and age, dietary pattern had an association with TG and TG/HDL-c in both groups. Compliance with this dietary pattern in the control group indicated a higher correlation with the cardio-metabolic risk in comparison with the PCOs group (Ehsani et al., 2016). The purpose of this study was to identify the VAI prediction model using a reduced rank regression. Moreover, it aimed to examine its associations with cardiometabolic risks in women with PCOs.

Table 1. Characteristics of included studies

\begin{tabular}{|c|c|c|c|}
\hline First author & $\begin{array}{l}\text { Study design, } \\
\text { Duration }\end{array}$ & Study population & Outcome \\
\hline $\begin{array}{l}\text { Foroozanfard, } \\
\text { et al. } 2017\end{array}$ & $\begin{array}{l}\mathrm{RCT}, 12 \\
\text { weeks }\end{array}$ & $\begin{array}{l}30 \text { DASH Group } \\
30 \text { control Group }\end{array}$ & $\begin{array}{l}\text { Significant reduction in BMI, AMH, MDA, HOMA-IR, } \\
\text { HOMA-B, but increase in QUICK, serum SHBG, NO in } \\
\text { DASH diet group. }\end{array}$ \\
\hline $\begin{array}{l}\text { Azadi-Yazdi, } \\
\text { et al. } 2016\end{array}$ & $\begin{array}{l}\text { RCT, } 3 \\
\text { months }\end{array}$ & $\begin{array}{l}60 \text { PCOS women } \\
30 \text { DASH group } \\
30 \text { control group }\end{array}$ & $\begin{array}{l}\text { Significant reduction in weight, BMI, fat mass and serum } \\
\text { androstenediones. Increase in SHBG and DPPH } \\
\text { scavenging activity in Dash compare to control group. }\end{array}$ \\
\hline $\begin{array}{l}\text { Asemi, et al. } \\
2015\end{array}$ & RCT, 8 weeks & $\begin{array}{l}48 \text { PCOs women , } \\
24 \text { DASH diet, } 24 \\
\text { control }\end{array}$ & $\begin{array}{l}\text { Significant reduction in waist, hip circumference, serum } \\
\text { insulin, HOMA-IR, serum hs-CRP in DASH diet group } \\
\text { and no significant differences in mean changes of FPG } \\
\text { and HOMA-B in both DASH and control groups. }\end{array}$ \\
\hline $\begin{array}{l}\text { Asemi, et al. } \\
2014\end{array}$ & RCT, 8 weeks & $\begin{array}{l}48 \text { PCOs women } \\
24 \text { DASH diet } \\
24 \text { control }\end{array}$ & $\begin{array}{l}\text { Significant reduction in weight, BMI, TG and VLDL-c } \\
\text { and significant increase in plasma total GSH and TAC in } \\
\text { DASH compare to control group. }\end{array}$ \\
\hline $\begin{array}{l}\text { Shishehgar, } \\
\text { et al. } 2016\end{array}$ & $\mathrm{CC}$ & $\begin{array}{l}142 \text { PCOs women } \\
140 \text { amenorrhea } \\
\text { non hirsute, BMI } \\
\text { and age matched }\end{array}$ & $\begin{array}{l}\text { Dietary pattern of PCOs women contain high glycemic } \\
\text { index food items and low legumes and vegetables }\end{array}$ \\
\hline $\begin{array}{l}\text { Ehsani, et al. } \\
2017\end{array}$ & $\mathrm{CC}$ & $\begin{array}{l}53 \text { PCOs women } \\
167 \text { age-matched } \\
\text { healthy women }\end{array}$ & $\begin{array}{l}\text { Consumption dietary pattern (high in fried vegetables, } \\
\text { vegetable oils except for olive oil and fast foods and low } \\
\text { consumption of dairy and cruciferous vegetables) had } \\
\text { significant association with higher systolic blood } \\
\text { pressure, WC, TC and LDL-c in control group. After } \\
\text { adjustment for age and BMI, dietary pattern was } \\
\text { associated with increase in TG and TG/HDL-c in both } \\
\text { groups. }\end{array}$ \\
\hline $\begin{array}{l}\text { Ahmadi, et al. } \\
2013\end{array}$ & $\mathrm{CC}$ & $\begin{array}{l}65 \text { PCOs women } \\
65 \text { age-matched } \\
\text { healthy women }\end{array}$ & $\begin{array}{l}\text { PCOs women consumed more calories and fat than } \\
\text { healthy women. No significant difference for BMI in two } \\
\text { groups, WC was significantly higher in the PCOs group. }\end{array}$ \\
\hline
\end{tabular}

DASH: Dietary approach stopped hypertension; RCT: Randomized clinical trial; CC: Case- control; PCOs: Polycystic ovary syndrome; BMI: Body mass index;AMH:Anti-mullerian; MDA: malondialdehyde; HOMA-IR: assessment-estimated insulin resistance; NO: Nitric oxide; FPG: Fasting plasma glucose; QUICK: Quantitative Insulin sensitivity Check index; SHBG: Sex hormone binding protein; DPPH: 2,2'-diphenyl-1-picryylhydrazyl; HOMA-IR: Homeostasis model of assessment insulin Resistance; hs-CRP: high-sensitivity C-reactive protein; HOMA-B: Homeostatic model assessment-beta cell function; TG: Triglycerides; VLDL-c: Very low-density lipoprotein; TAC: Total antioxidant capacityGSH: Total glutathione; WC: Waist circumference; TC: Total cholesterol; LDL-c: Low-density lipoprotein; HDL-c: High-density lipoprotein 


\section{Discussion}

The researchers concluded that some of the dietary patterns might be appropriate to treat PCOs. In recent studies, some healthy diets such as DASH diet were prescribed to the PCOs women. DASH diet is a low-glycemic-index low-energy dense diet rich in fruits, vegetables, whole grains, and low-fat dairy products, but low in saturated fats, cholesterol, refined grains, and sweets. This eating plan was initially proposed for the management of hypertension (Vollmer et al., 2001). It also showed beneficial effects on type 2 diabetes (Azadbakht et al., 2011), gestational diabetes (Asemi et al., 2013), inflammatory factors (Soltani et al., 2016), metabolic syndrome (Esposito and Giugliano, 2006), and non-alcoholic fatty liver disease (NAFLD) (Razavi Zade et al., 2016). This dietary pattern contains high amounts of dietary fiber, phytoestrogens, antioxidants, isoflavones, magnesium, and calcium, along with its LGI (Ahmadi et al., 2013) . Regarding this diet, antioxidants and magnesium intake were inversely associated with insulin resistance (Puchau et al., 2010). Moreover, DASH diet contains high antioxidants and arginine amino acids that may improve metabolic status using the decreased NADPH oxidase activity and reduced level of angiotensin II (Chen et al., 2001). On the other hand, increased intake of micronutrients, such as calcium in DASH diet would result in fecal fat excrete, weight loss, and increased insulin

\section{References}

Ahmadi A, et al. 2013. Anthropometric characteristics and dietary pattern of women with polycystic ovary syndrome. Indian journal of endocrinology and metabolism. 17 (4): 672-676.

Amr N \& Abdel-Rahim HE 2015. The effect of chromium supplementation on polycystic ovary syndrome in adolescents. Journal of pediatric and adolescent gynecology. 28 (2): 114-118.

Asemi Z \& Esmaillzadeh A 2015. DASH diet, insulin resistance, and serum hs-CRP in polycystic ovary syndrome: a randomized controlled clinical trial. Hormone and metabolic research. 47 (3): 232-238. sensitivity (Onakpoya et al., 2011). Furthermore, weight reduction might reduce testosterone levels (Luo and $\mathrm{Xu}, 2012$ ). Nevertheless, this dietary pattern seems to be potentially beneficial for women with PCOS.

In recent years, very limited number of studies was conducted to find an appropriate dietary pattern for women with PCOS. So, further studies are needed to investigate the effective dietary patterns in PCOS women for treatment and reduction of this syndrome and its complications.

\section{Conclusion}

Studies concluded that diet modification such as DASH diet, increase of the protein/carbohydrate ratio in the diet, the low calorie diets, or iso-caloric diets with a low glycemic index may have beneficial effects in treatment of women with PCOS. However, studies which conducted on finding appropriate dietary pattern for this syndrome are limited and Future Studies are required to finding major dietary pattern in women with PCOS.

\section{Authors' contribution}

All authors participated to designing, developing the search strategy and writing the manuscript. Panjeshahin A and Khosravi $M$ conducted the systematic search and study selection; all authors read and approved the final manuscript.

\section{Conflict of interest}

There is not any conflict of interest.

Asemi Z, et al. 2014. Effects of DASH diet on lipid profiles and biomarkers of oxidative stress in overweight and obese women with polycystic ovary syndrome: A randomized clinical trial. Nutrition. 30 (11-12): 1287-1293.

Asemi Z, Tabassi Z, Samimi M, Fahiminejad T \& Esmaillzadeh A 2013. Favourable effects of the Dietary Approaches to Stop Hypertension diet on glucose tolerance and lipid profiles in gestational diabetes: a randomised clinical trial. British journal of nutrition. 109 (11): 2024-2030.

Azadbakht L, et al. 2011. Effects of the Dietary Approaches to Stop Hypertension (DASH) 
eating plan on cardiovascular risks among type 2 diabetic patients: a randomized crossover clinical trial. Diabetes care. 34 (1): 55-57.

Azadi-Yazdi M, Karimi-Zarchi M, SalehiAbargouei A, Fallahzadeh H \& Nadjarzadeh A 2017. Effects of Dietary Approach to Stop Hypertension diet on androgens, antioxidant status and body composition in overweight and obese women with polycystic ovary syndrome: a randomised controlled trial. Journal of human nutrition and dietetics. 30 (3): 275283.

Carmina E, Guastella E \& Longo RA 2016. Advances in the diagnosis and treatment of PCOs. Current pharmaceutical design. 22 (36): 5508-5514.

Chen X, Touyz RM, Park JB \& Schiffrin EL 2001. Antioxidant effects of vitamins $C$ and $E$ are associated with altered activation of vascular NADPH oxidase and superoxide dismutase in stroke-prone SHR. Hypertension. 38 (3): 606611.

Cree-Green M 2017. Worldwide Dissatisfaction With the Diagnostic Process and Initial Treatment of PCOS. Journal of clinical endocrinology \& metabolism. 102 (2): 375-378.

Ehsani B, et al. 2016. A visceral adiposity indexrelated dietary pattern and the cardiometabolic profiles in women with polycystic ovary syndrome. Clinical nutrition. 35 (5): 1181-1187.

Faghfoori Z, Fazelian S, Shadnoush M \& Goodarzi R 2017. Nutritional management in women with polycystic ovary syndrome: A review study. Diabetes \& metabolic syndrome. 11: S429-432.

Farshchi H, Rane A, Love A \& Kennedy RL 2007. Diet and nutrition in polycystic ovary syndrome (PCOS): pointers for nutritional management. Journal of obstetrics and gynaecology 27 (8): 762-773.

Foroozanfard F, et al. 2017. The effects of dietary approaches to stop hypertension diet on weight loss, anti-Mullerian hormone and metabolic profiles in women with polycystic ovary syndrome: A randomized clinical trial. Clinical endocrinology. 87 (1): 51-58.
Goodarzi MO, Dumesic DA, Chazenbalk G \& Azziz R 2011. Polycystic ovary syndrome: etiology, pathogenesis and diagnosis. Nature reviews endocrinology. 7 (4): 219-231.

Jalilian A, et al. 2015. Prevalence of polycystic ovary syndrome and its associated complications in Iranian women: A meta-analysis. Iranian journal of reproductive medicine. 13 (10): 591604.

Kalem MN, Kalem Z \& Gurgan T 2017. Effect of metformin and oral contraceptives on polycystic ovary syndrome and IVF cycles. Journal of endocrinological investigation. 40 (7): 745-752.

Love JG, et al. 2016. The experiences of women with polycystic ovary syndrome on a very lowcalorie diet. Internationa journal womens health. 8: 299-310.

Luo X \& Xu L 2012. Association of fat distribution with metabolic syndrome in patients with polycystic ovary syndrome. Journal of southern medical University. 32 (9): 1325-1327.

Onakpoya IJ, Perry R, Zhang J \& Ernst E 2011. Efficacy of calcium supplementation for management of overweight and obesity: systematic review of randomized clinical trials. Nutrition reviews. 69 (6): 335-343.

Palomba S, Santagni S, Falbo A \& La Sala GB 2015. Complications and challenges associated with polycystic ovary syndrome: current perspectives. International journal womens health. 7: 745-763.

Pastore LM, Patrie JT, Morris WL, Dalal P \& Bray MJ 2011. Depression symptoms and body dissatisfaction association among polycystic ovary syndrome women. Journal of psychosomatic research. 71 (4): 270-276.

Puchau B, Zulet MA, de Echávarri AG, Hermsdorff HHM \& Martínez JA 2010. Dietary total antioxidant capacity is negatively associated with some metabolic syndrome features in healthy young adults. Nutrition. 26 (5): 534-541.

Razavi Zade M, et al. 2016. The effects of DASH diet on weight loss and metabolic status in adults with non- alcoholic fatty liver disease: a 
randomized clinical trial. Liver international. $\mathbf{3 6}$ (4): 563-571.

Shishehgar F, et al. 2016. Comparison of dietary intake between polycystic ovary syndrome women and controls. Global journal of health science. 8 (9): 54801.

Soltani S, Shirani F, Chitsazi MJ \& SalehiAbargouei A 2016. The effect of dietary approaches to stop hypertension (DASH) diet on weight and body composition in adults: a systematic review and meta- analysis of randomized controlled clinical trials. Obesity reviews. 17 (5): 442-454.
Sorensen LB, Soe M, Halkier KH, Stigsby B \& Astrup A 2012. Effects of increased dietary protein-to-carbohydrate ratios in women with polycystic ovary syndrome. American journal clinical nutrition. 95 (1): 39-48.

Vollmer WM, et al. 2001. Effects of diet and sodium intake on blood pressure: subgroup analysis of the DASH-sodium trial. Annals of internal medicine. 135 (12): 1019-1028.

Wang ET, et al. 2011. Polycystic ovary syndrome and risk for long-term diabetes and dyslipidemia. Obstetrics \& gynecology. 117 (1): 6-13. 